\title{
GUNN DOMAIN EXISTENCE IN THE CHANNEL OF A SATURATED GaAs MESFET
}

\author{
P. BONJOUR, R. CASTAGNE and J. P. COURAT
}

Institut d'Electronique Fondamentale (*), Université de Paris-Sud, 91405 Orsay Cedex, France

\begin{abstract}
Résumé. - L'existence d'un domaine de Gunn dans le canal d'un MESFET GaAs après saturation est confirmée par une simulation numérique bidimensionnelle, mais son apparition ne produit pas de décroissance du courant de drain. On montre que dans les dispositifs comportant une zone de charge d'espace à l'interface entre couche et substrat la zone d'accumulation du domaine provoque un élargissement du canal responsable de la circulation d'un courant excédentaire, sans que le substrat soit sollicité.
\end{abstract}

\begin{abstract}
The existence of a Gunn domain in the channel of a GaAs MESFET after saturation is confirmed through numerical bidimensional simulation, but the $i_{\mathrm{D}} v s$. $V_{\mathrm{DS}}$ decrease is not observed. It is shown that in devices including an interfacial space charge layer, the accumulation bump of the domain is responsible for an opening of the channel by which the leakage current is flowing.
\end{abstract}

1. Introduction. - Drain conductance of the GaAs MESFET in the saturation range is a very important parameter. However its calculation is made difficult because of the highly non linear velocity field characteristics in $\mathrm{GaAs}$ and the bidimensional character of the conduction phenomenon in the FET.

In most cases, the drain current $i_{\mathrm{D}} v s$ drain to source voltage $v_{\mathrm{DS}}$ of short channel GaAs MESFET, experimentally observed, shows a slight increase of $i_{\mathrm{D}}$ beyond saturation. On the other hand, both numerical and analytical models [1-4] of the intrinsic device (i.e. without a substrate) show that the carrier velocity limitation appears together with a stationary Gunn domain responsible for a decrease in the drain current. The interpretation usually admitted $[1,5]$ for the observed excess saturation current is that of a leakage path through the substrate, but in order to explain this excess current, the concentration of mobile carriers in the substrate must be supposed significantly higher than the intrinsic concentration. On the other hand, many experimental results [6-8] give a strong evidence of an interfacial space charge layer due to trapping phenomenons, the result being that the carriers in the active layer can hardly penetrate into the substrate. The fact that devices including such interfacial space charge layer (SCL) no longer exhibit a decrease of their drain current after saturation has led us to study this problem. The purpose of this paper is then to give a qualitative insight into the behaviour of the Gunn domain and of the excess saturation current, using one-dimensional and bidimensional simulation of short channel GaAs FET.

2. One dimensional model and the Gunn effect. A fast one-dimensional numerical model has been

(*) G.R.E.C.O., Micro-ondes, CNRS nº 11 used to describe the intrinsic FET (i.e. without a substrate). In this model the channel is considered as a current tube whose cross-section is made to vary from drain to source in order to take into account the gate SCL penetration. The later is computed under the total depletion approximation using the potential difference between the gate and the centre of one cell in the channel. Edge effects are considered by shaping the SCL beyond the gate as a quarter of an ellipse [9]. This model includes the static velocity vs field characteristic [3, 4] and solves simultaneously the Poisson's and conservation equations.

We have simulated the intrinsic part of the FET described in table I. If the low field mobility is higher than $2000 \mathrm{~cm}^{2} \mathrm{~V}^{-1} \mathrm{~s}^{-1}$ a stationary Gunn domain appears (Fig. 2a) and simultaneously a substantial decrease in the drain current is observed after saturation (Fig. 2b). Such a behaviour was found whatever the active layer thickness is in intrinsic FET. This shows that the excess current mechanism is to be found in bidimensional effects.

\section{TABLE I}

\section{Characteristics of the studied devices}

Active layer doping concentration Substrate doping concentration Active layer thickness

Substrate thickness

Low field mobilities in active layer in substrate

Critical field for velocity saturation

Saturated velocity value

Length of the source or drain metallized contacts

Gate to drain space

$2 \times 10^{16} \mathrm{~cm}^{-3}$

$1 \times 10^{12} \mathrm{~cm}^{-3}$

$2500 \AA$

$6000 \AA$

$3000 \mathrm{~cm}^{2} \mathrm{~V}^{-1} \mathrm{~s}^{-1}$

$5000 \mathrm{~cm}^{2} \mathrm{~V}^{-1} \mathrm{~s}^{-1}$

$3950 \mathrm{~V} \mathrm{~cm}^{-1}$

$8 \times 10^{6} \mathrm{~cm} \mathrm{~s}^{-1}$

First geometry : gate length $1 \mu \mathrm{m}$, source to gate space $1 \mu \mathrm{m}$.

Second geometry : gate length $0.3 \mu \mathrm{m}$, source to gate space $0.3 \mu \mathrm{m}$. 
3. Bidimensional model and by-passing of the Gunn domain. - A two dimensional numerical model for static or dynamic computation has been written $\left({ }^{1}\right)$. The Laplace-Poisson's and conservation equations are solved by a classical finite difference method with a specially fast algorithm including the static velocity vs field characteristics. This program can accept every geometry or doping profiles. Boundary values are given for both the carrier concentration $n$ and the potential $V$ under ohmic contacts. Everywhere else, zero normal derivatives are assumed. The performances of this program are satisfactory. It takes for instance $20^{\prime}$ on Univac 1110 computer to cal-

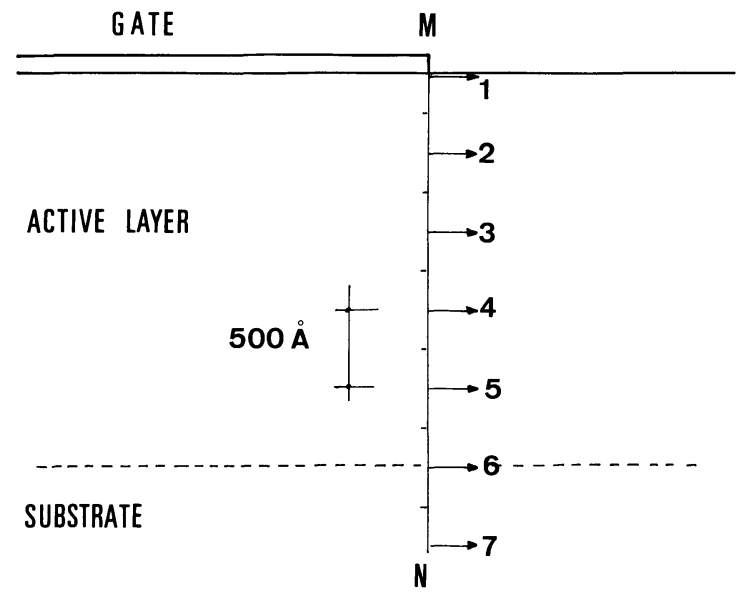

FIG. 1.

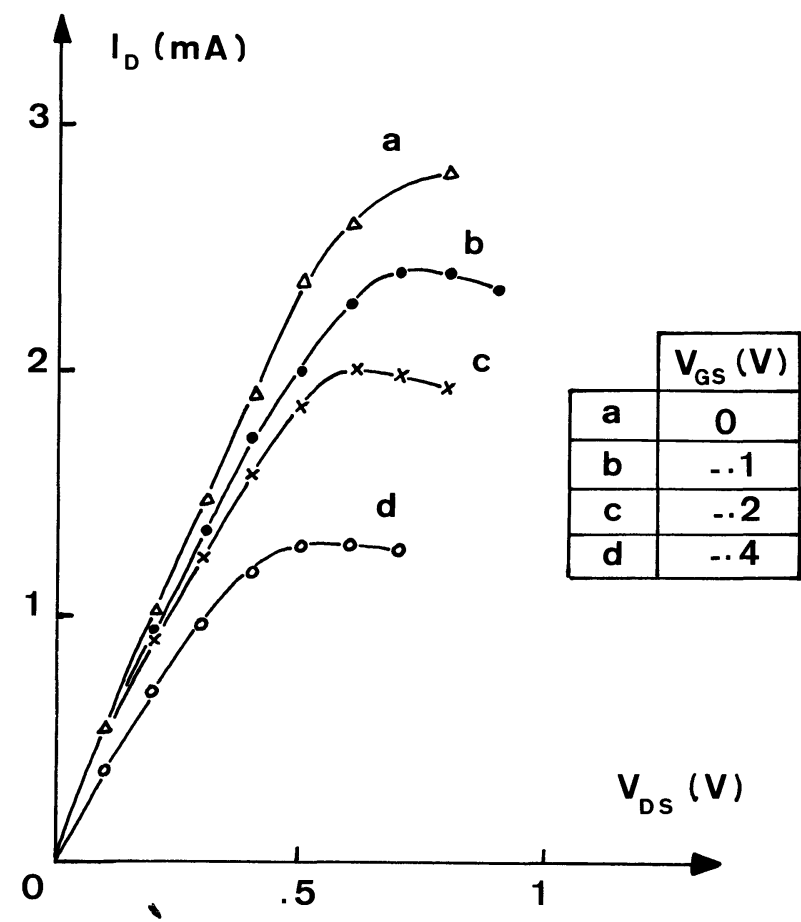

FIG. 2. - Drain characteristics of the intrinsic part of the FET described in table I (one dimensional modelling).

( $\left.{ }^{1}\right)$ The elaboration of the program was supported by the DGRST by decision 77.70983 of the CCM Committee. culate 12 points of the static $i_{\mathrm{D}} / v_{\mathrm{Ds}}$ characteristics for one of the devices described in figure 1 . We have considered two types of interfaces between the active layer and the substrate. The first one is a HL junction with a concentration decrease of one decade about $800 \AA$ under the epilayer, followed by a $\mathrm{n}$ type uniformly doped $\left(10^{12} \mathrm{~cm}^{-3}\right)$ substrate. The second one is an interfacial barrier including a fixed negatively charged layer with an apparent surface charge density of $10^{11} \mathrm{~cm}^{-2}$ which is responsible for the creation of a depleted region between the active layer and the substrate. We have also considered two sets of dimensions for the gate and for the gate to source interval as described in table I. In order to study the channel distorsion more easily, we will give the results obtained for a very short channel in detail, and merely mention the results obtained with the other geometry.

Devices with HL interface exhibit a significative permanent injection in the substrate due to the diffusion from the highly doped layer. But this effect is artificially reinforced by a mirror effect introduced by the Neumann boundary condition used at the bottom of the substrate. Figure 3 shows the equiconcentration curves and figure 4 the electron flux

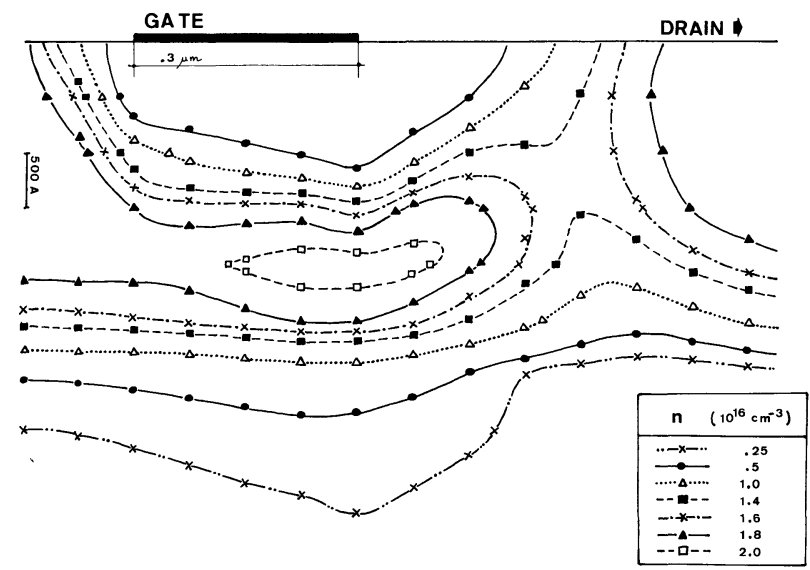

FIG. 3. - Equidensity curves in HL interface FET $(0.3 \mu \mathrm{m}$ gate) for $V_{\mathrm{DS}}=1.2 \mathrm{~V}$ and $-V_{\mathrm{GS}}+V_{\mathrm{B}}=0.1 \mathrm{~V}$.

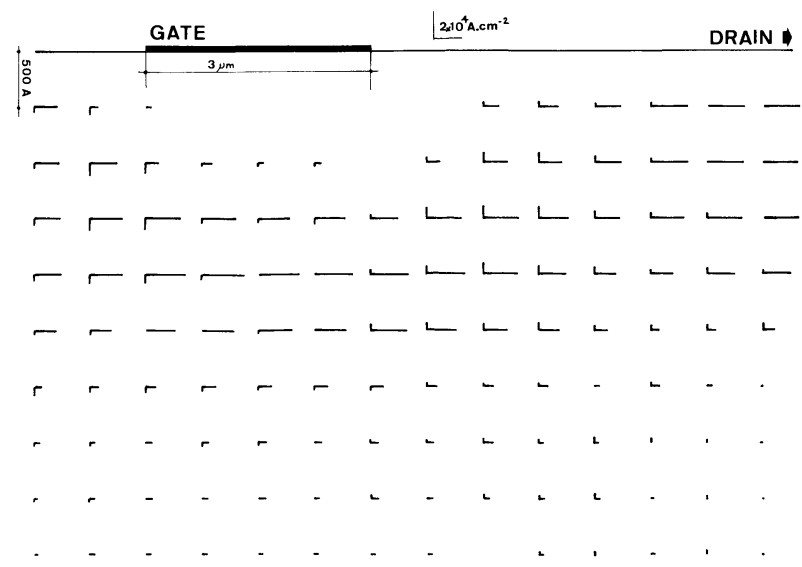

Fig. 4. - Electron flux vector components in $\mathrm{HL}$ interface FET $(0.3 \mu \mathrm{m}$ gate $)$ for $V_{\mathrm{DS}}=1.2 \mathrm{~V}$ and $-V_{\mathrm{GS}}+V_{\mathrm{B}}=0.1 \mathrm{~V}$. 
density distribution for a gate diffusion potential $-V_{\mathrm{GS}}+V_{\mathrm{B}}=0.1 \mathrm{~V}$ and $V_{\mathrm{DS}}=1.2 \mathrm{~V}$. Current is flowing in the interfacial part of the device under the gate, showing the commonly predicted leakage path. Figure 5 shows the $i_{\mathrm{D}} v s . v_{\mathrm{Ds}}$ characteristics : curves 1 for $0.3 \mu \mathrm{m}$ gate, curve 2 for $1 \mu \mathrm{m}$ gate.

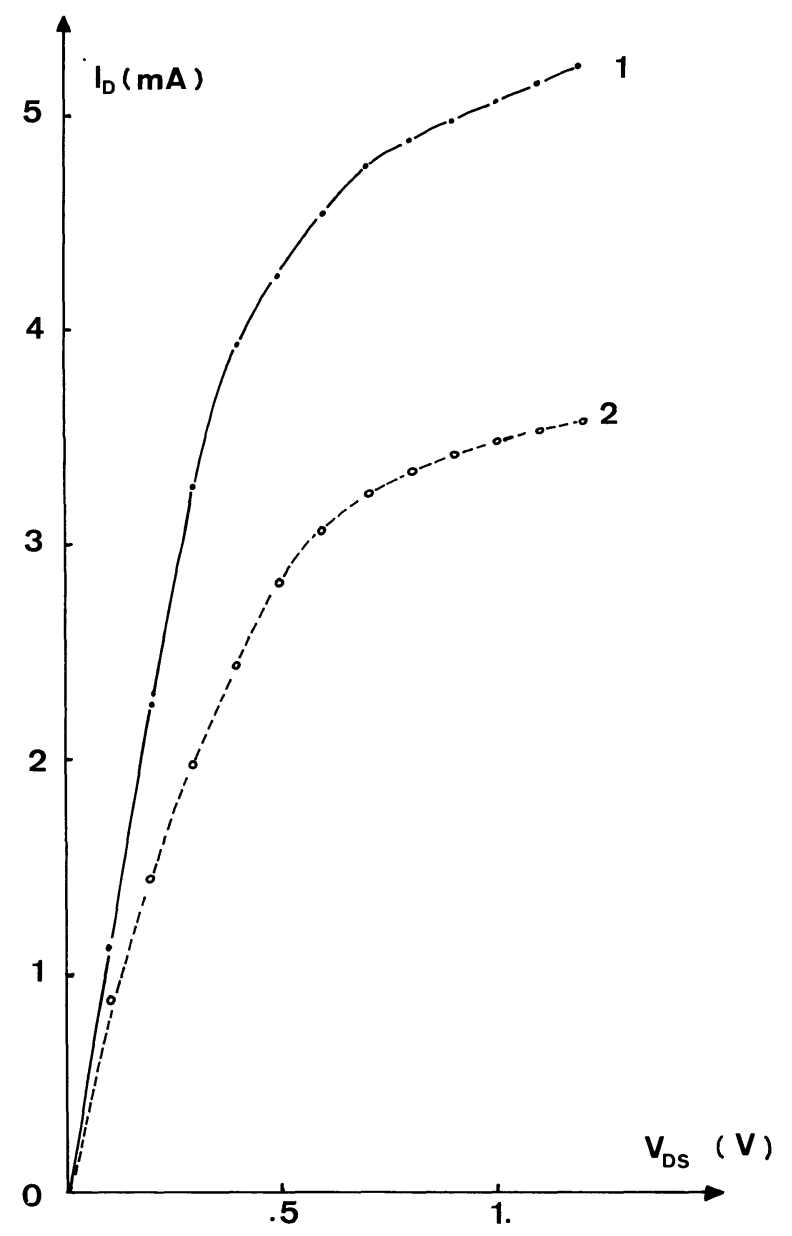

FIG. 5. - Drain characteristics of HL interface FET curve 1 : $0.3 \mu \mathrm{m}$ gate, curve $2: 1 \mu \mathrm{m}$ gate.

In devices with an interfacial space charge layer which we believe more representative of the actual devices - and for $-V_{\mathrm{GS}}+V_{\mathrm{B}}=0.1 \mathrm{~V}$ and $V_{\mathrm{DS}}=0$, the electron density in the channel is $1.6 \times 10^{16} \mathrm{~cm}^{-3}$ below the doping level i.e. the device is normally off. Figure 6 shows $i_{\mathrm{D}} / v_{\mathrm{DS}}$ for $-V_{\mathrm{GS}}+V_{\mathrm{B}}=0.1 \mathrm{~V}$. The currents flowing through the vertical plane named $\mathrm{MN}$ in figure 1 , at levels named 1 to 8 are also plotted. The variations of these currents with $v_{\mathrm{DS}}$ give informations about the path followed by the electrons when saturation is developed. The $i_{\mathrm{D}} / v_{\mathrm{DS}}$ curve for the $1 \mu \mathrm{m}$ geometry device is also given in figure 6 showing, by comparison, that the excess saturation current is strongly reinforced by the gate width reduction.

Figures 7 to 10 show equal density curves and electron flux density distribution respectively for $V_{\mathrm{DS}}=0.4 \mathrm{~V}$ and $V_{\mathrm{DS}}=1.2 \mathrm{~V}$. The following points are to be noticed.

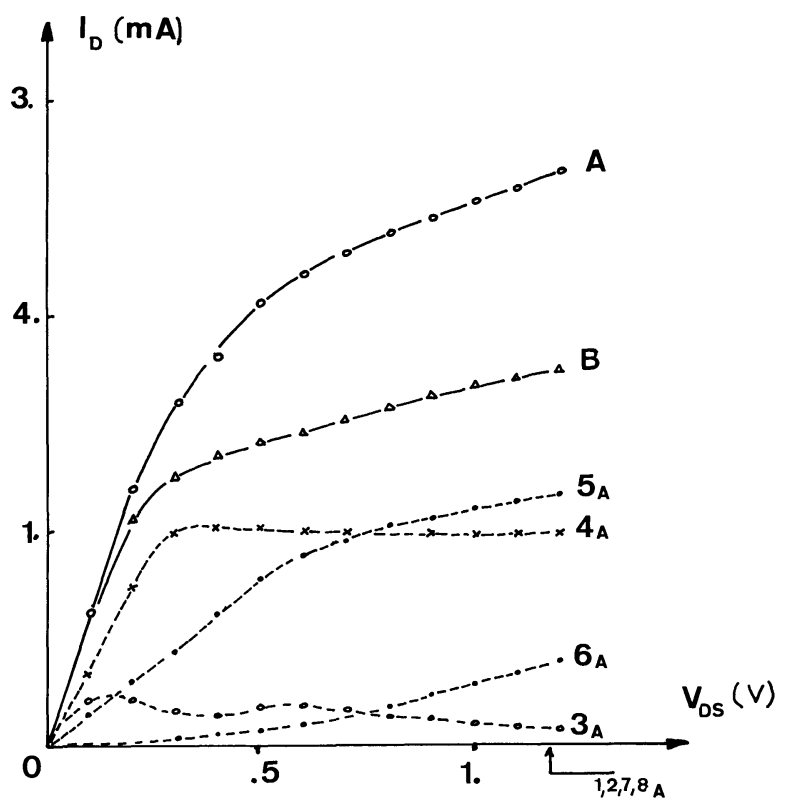

FIG. 6. - Drain characteristics of FET including an interfacial space charge layer : curve $A i_{\mathrm{D}}$ vs. $V_{\mathrm{DS}} 0.3 \mu \mathrm{m}$ gate ; curve $3_{\mathrm{A}}, 4_{\mathrm{A}}$, $5_{A}, 6_{A}$ currents flowing respectively in cross-sections referenced in figure 1 ; curve $\mathrm{B} i_{\mathrm{D}} v s . V_{\mathrm{DS}} 1 \mu \mathrm{m}$ gate.

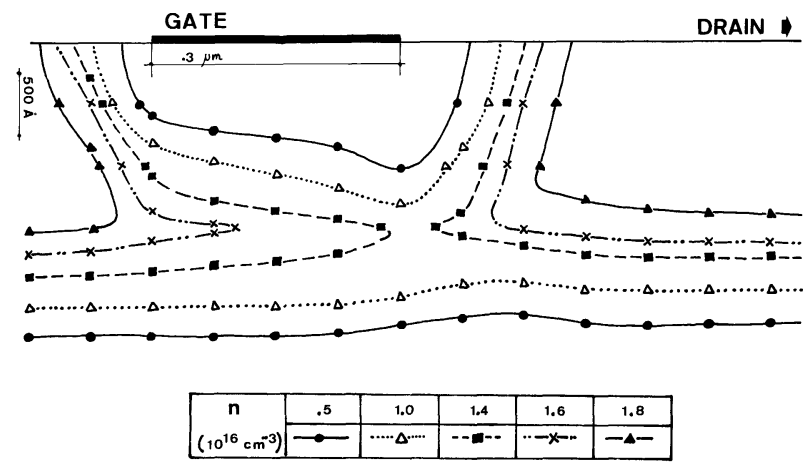

FIG. 7. - Equidensity curves in SCL interface FET $(0.3 \mu \mathrm{m}$ gate) for $V_{\mathrm{DS}}=0.4 \mathrm{~V}$ and $-V_{\mathrm{GS}}+V_{\mathrm{B}}=0.1 \mathrm{~V}$.

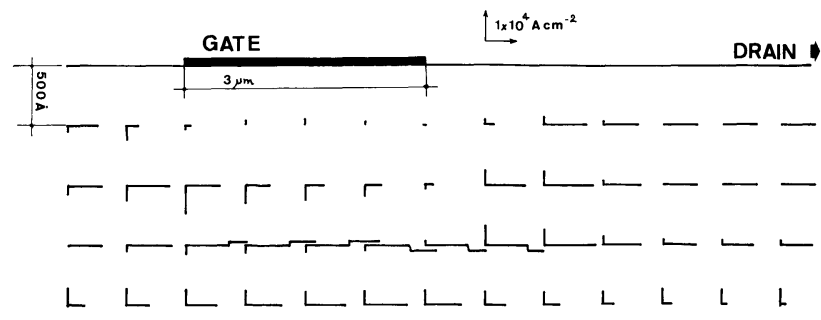

FIG. 8. - Electron flux vector components in SCL interface FET $\left(0.3 \mu \mathrm{m}\right.$ gate) for $V_{\mathrm{DS}}=0.4 \mathrm{~V}$ and $-V_{\mathrm{GS}}+V_{\mathrm{B}}=0.1 \mathrm{~V}$.

1) At $V_{\mathrm{DS}}=0.4 \mathrm{~V}$, the channel is pinched under the end of the gate. Carrier density has fallen down to $10^{16}$ and the electric field has already reached the critical value.

At $V_{\mathrm{DS}}=1.2 \mathrm{~V}$, the carrier injection from the source has been replaced by the accumulation bump 


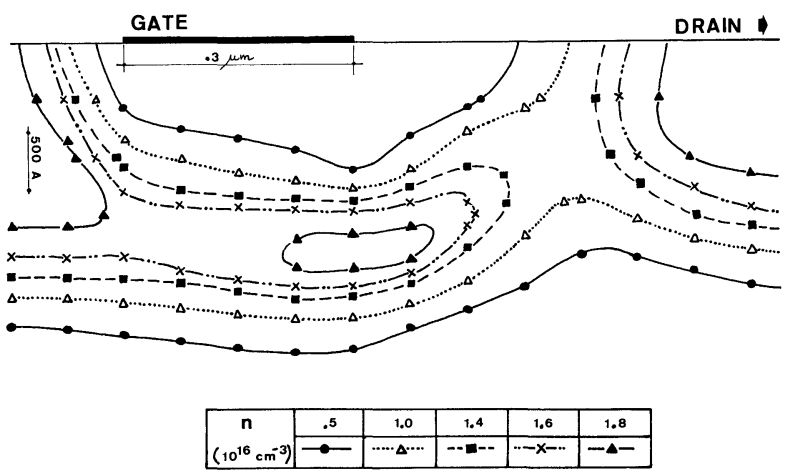

FIG. 9. - Equidensity curves in SCL interface FET $(0.3 \mu \mathrm{m}$ gate $)$ for $V_{\mathrm{DS}}=1.2 \mathrm{~V}$ and $-V_{\mathrm{GS}}+V_{\mathrm{B}}=0.1 \mathrm{~V}$.

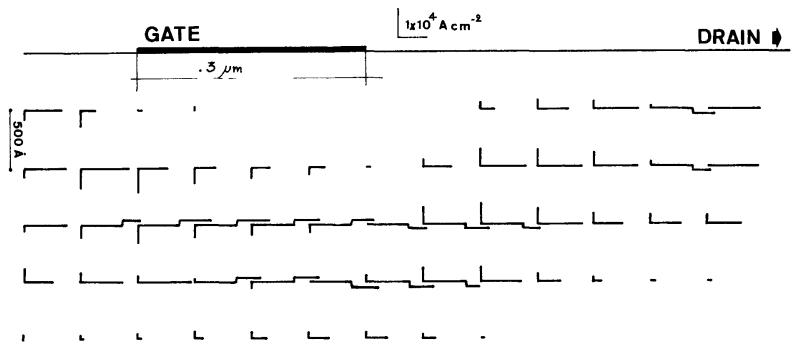

FIG. 10. - Electron flux vector components in SCL interface FET $\left(0.3 \mu \mathrm{m}\right.$ gate) for $V_{\mathrm{DS}}=1.2 \mathrm{~V}$ and $-V_{\mathrm{GS}}+V_{\mathrm{B}}=0.1 \mathrm{~V}$.

of what can be considered as a stationary Gunn domain trapped under the gate.

2) Beyond the gate end, the interfacial SCL deeply penetrates towards the drain because of the drain bias and the Gunn domain depletion layer. The channel passes round the obstacle towards the surface and is consequently strongly distorted. The extension of the interfacial SCL under the influence of the drain bias in that region known to be rich in trapping centres could be the cause of the experimentally observed hysteresis phenomenon on $i_{\mathrm{D}} v s . v_{\mathrm{DS}}$ curves.

3) The domain accumulation layer appears together with an opening of the channel by pushing away both gate and interfacial space charge layer. This offers a leakage path for the drain current. Figure 6 clearly shows that currents numbered 5 and 6 are responsible for the excess saturation current and figures 1,9 show that these currents flow between the accumulation region of the Gunn domain and the interfacial space charge layer. No current is flowing through the substrate.

4. Conclusion. - The existence of a Gunn domain in the channel of the GaAs FET after saturation is then confirmed, though the $i_{\mathrm{D}} v s$. $v_{\mathrm{DS}}$ curve $\mathrm{c}$ does not exhibit the characteristic of $i_{\mathrm{D}}$. That is true of HL interface as well as for SCL interface. In this late situation however, in spite of the impossibility for mobile carriers to penetrate the substrate, a leakage is nevertheless created through a distorsion and an opening of the channel. In such a situation the domain position is a very important parameter and it is a reason why the velocity overshoot phenomena could play an important role in the saturation mechanism.

\section{References}

[1] Engelmann, R. W. H. and Liechti, C. A., Digest of the International Electron Devices Meeting Palo Alto 1976, p. 351.

[2] Shur, M. S., Eastman, L. F., IEEE Trans ED 25 (1978).

[3] Himsworth, B., Solid State Electron. 15 (1972) 1353.

[4] Yamaguchi, K. et al., IEEE Trans ED 23 (1976).

[5] Reiser, M., Electron. Lett. 6 (1970) 493.
[6] Tranduc, H. et al., Revue Phys. Appl. 13 (1978).

[7] Houng, Y. M. and Pearson, G. L., J. Appl. Phys. 49 (1978) 3348.

[8] TANimoto et al., 6th ESSDERC Série B4, Munich 1976.

[9] Jun'ichi SONE, Takayama, Electron. Lett. 12 (1976) 622. 